\title{
Comments on "Conceptions are Social Constructs" by H. v. Braun, W. Hesse, U. Andelfinger, H.-B. Kittlaus and G. Scheschonk
}

\author{
Hannu Kangassalo \\ University of Tampere, PO Box 607, FIN-33101 Finland \\ hk@acs.uta.fi
}

The FRISCO report aims to form a comprising and consistent system of terms and concepts which could be applied to handle issues, problems and solutions in the information systems field. The overall approach used is based on language and semiotics illustrated by the use of a variant of the semiotic triangle extended by adding an actor at its central position.

In more general terms, the report concerns the notions, views, and rules planned to be used for designing, understanding, and evaluating information systems, i.e. the notions, views, and rules for description, subsumption, explanation, prediction, and retrodiction of information available for describing the domain and informational constructs deducible from it. The issues in the report concern partly with the nature of knowledge and the structure of it, and possibility, scope, and general basis of knowledge. They also deal with a question on which grounds knowledge about the domain, views, concepts, rules, and facts in information systems are based, and which kind of justification we can have for that knowledge.

In the paper of "Conceptions are social Constructs - ..." the authors analyse the use of the (tetrahedron) variant of the semiotic triangle. They concentrate on the notion of conception which they recognise to be important but problematic. They propose that the interpretation of the notion of conception should be revised. 
First, the notion of conception should rather be based on cognition of an observer than on psychological disposition of perception. Second, they give an explanation of the notion of conception which is operational in the sense that a given interpretation of a representation can be reconstructed or even proved to be correct or not. Third, they propose that the term thing might be identified with the term conception. Fourth, they reveal some deficiencies and circular constructs in the structure of the concept system presented in the report, and they propose how these "problematic" constructs could be corrected or removed.

In information systems design we are constructing models about the domain, and knowledge based on them. This approach turns our point of view into issues of scientific methodology; how can one develop models or theories that are better than competing models or theories? That question is a fundamental problem in the area of theory of knowledge or epistemology. We have to pay more attention to the epistemology of information systems, which is a branch of research that studies knowledge in information systems, especially questions, e.g. how that knowledge is or should be synthesised, and how can one develop models or theories that are better than competing models or theories?

There are different approaches to epistemology of information systems. These approaches are not necessarily coherent with each other, and it may be that they can not be harmonised into one system of concepts to form a coherent theory. In the FRISCO report the main part of the work is based on one epistemology, but there is also a dissenting position expressed which indicates that a quite different ontology and epistemology could be applied, too. Also the authors of the paper of "Conceptions are social Constructs - ..." seem to support some of the views in this other epistemology. Research on this direction should be continued, and also other possible epistemologies should be searched for.

The main epistemological approach in the report is constructivism, about which there are different branches, e.g. individual constructivism and social constructivism. These branches are not recognised in the report. The first one assumes that an individual attempts to reach coherence among the different pieces of knowledge. The second one sees consensus between different subjects as the ultimate criterion to judge knowledge. 'Truth' will be accorded only to those constructions on which most people of a social group agree. 
The authors say that conceptions as "social constructs" are objective and operational, i.e. verifiable or at least reconstructable by applying laws and rules [see abstract]. That is an ideal, but very often constructs seem to be more or less biased, on the basis of erroneous assumptions of the group. Later the authors discuss about observers and say that "essential for the flexibility of the whole approach is that we can consider individual observers as well as groups or even whole societies." That is true, but from it follows that both individual constructivism and social constructivism must explicitly be taken into account. That has not been done. All individuals have originally their own conceptions, and even in communication situations the receiver tries to understand an incoming message from his own point of view. There are methods how communicating parties may try to improve their mutual understanding but complete understanding can hardly be achieved. Learning and extensive education systems tend to standardise some of the conceptions, but not all of them. The authors also emphasise that conceptions are a product of social agreement and they may vary if such agreements change in time. However, in the analysis of changes the identity of conceptions and naming relations used in the language must be taken into account, too.

The authors say also that "The intuitively used construction rules and intuitively applied construction acts can be made explicit by pointing out the logic of the relations existing between terms, between referents, and between terms and referents of a proposition". They propose a logic of construction acts. Is it the same thing than a conceptualisation process in formal ontology?

It seems that authors' proposals direct the work into a right direction, but the research on deep ontological and epistemological aspects of information systems should be continued, especially on applied principles of information system epistemology, formal ontology, and construction and use of conceptions and also specific concepts. 